\title{
Testing the Quality of Manufactured Disks and Balls*
}

\author{
Prosenjit Bose and Pat Morin \\ School of Computer Science, Carleton University, Ottawa, CANADA, K1S 5B6 \\ \{morin, jit\}@cs.carleton.ca
}

\begin{abstract}
We consider the problem of testing the roundness of manufactured disks and balls using the finger probing model of Cole and Yap [6]. The running time of our procedures depends on the quality of the object being considered. Quality is a parameter that is negative when the object is not sufficiently round and positive when it is. Quality values close to 0 represent objects that are close to the boundary between sufficiently round and insufficiently round.

When the object being tested is a disk and its center is known, we describe a procedure that uses $O(n)$ probes and $O(n)$ computation time. (Here $n=|1 / q|$, where $q$ is the quality of the object.) When the center of the object is not known, a procedure using $O(n)$ probes and $O(n \log n)$ computation time is described. When the object is a ball, we describe a procedure that requires $O\left(n^{2}\right)$ probes and $O\left(n^{4}\right)$ computation time. Lower bounds are also given that show that these procedures are optimal in terms of the number of probes used. These results extend previous results in two directions by relaxing some of the assumptions required by previous results and by extending these results for 3-dimensional objects.
\end{abstract}

\section{Introduction}

The field of metrology is concerned with measuring the quality of manufactured objects. A basic task in metrology is that of determining whether a given manufactured object is of acceptable quality. Usually this involves probing the surface of the object using a measuring device such as a coordinate measuring machine to get a set $S$ of sample points, and then verifying, algorithmically, how well $S$ approximates an ideal object.

\footnotetext{
${ }^{*}$ This work was funded in part by the Natural Sciences and Engineering Research Council of Canada
} 
A special case of this problem is determining whether an object is round. For our purposes, an object $I$ is good if the boundary of $I$ can be contained in an annulus of inner radius $1-\epsilon$ and outer radius $1+\epsilon$, for some quality parameter $\epsilon>0$, and is bad otherwise. See Figure 1 for examples of good and bad objects. We call this problem the roundness classification problem.

In the field of computational geometry a lot of work has been done on finding efficient algorithms for testing the roundness of a set of sample points, using the definition of roundness given above $[4,7,13]$, as well as several other definitions of roundness $[2,3,1,5,8,9,10,11,15,14,16,18]$.

However, very little research has been done on probing strategies for the roundness classification problem. A notable exception is the work by Mehlhorn, Shermer, and Yap [12, 17], in which a probing strategy for manufactured disks is coupled with a roundness testing algorithm. Unfortunately, the procedure described in $[12,17]$ relies on the assumption that the object $I$ is convex. It is usually not the case that the manufacturing process can guarantee this.

In this paper we describe strategies for testing the roundness of manufactured disks and balls. We use the finger probing model of Cole and Yap [6]. In this model, the measurement device can identify a point in the interior of $I$ and can probe along any ray originating outside of $I$, i.e., determine the first point on the ray that intersects the boundary of $I$ (see Figure 2). The finger probing model is a reasonable abstract model of a coordinate measuring machine or a laser rangefinder [19]. A coordinate measuring machine consists of a robotic arm with a pressure sensitive tip that determines points on the surface of an object by poking it. A laser range finger is a device that records points on the surface of an object by shooting a laser at it.

This work extends the results of Mehlhorn et al $[12,17]$ in several ways. The assumption that the object $I$ is convex is replaced by a much weaker assumption related to visibility. Using this assumption, we give a procedure for testing the roundness of a manufactured disk $I$ using $O(n)$ probes and $O(n \log n)$ computation time. Here $n=1 / \mid$ qual $(I) \mid$ where qual $(I)$ measures how far the object $I$ is from the boundary between good and bad. This matches the number of probes and computation time required by Mehlhorn et al for convex objects. For testing the roundness of manufactured balls, we give an algorithm that uses $O\left(n^{2}\right)$ probes and $O\left(n^{4}\right)$ computation time. This is the first result testing the quality of 3-dimensional objects using finger probes. We also show that all our results our optimal in terms of the number of probes.

The remainder of the paper is organized as follows: Section 2 introduces definitions and notation used throughout the remainder of the paper. Section 3 describes procedures for testing the quality of manufactured disks. Section 4 describes procedures for testing the quality of manufactured balls. 


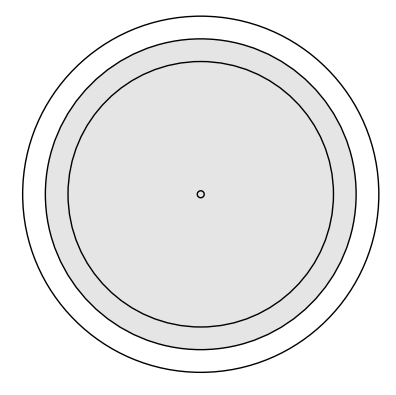




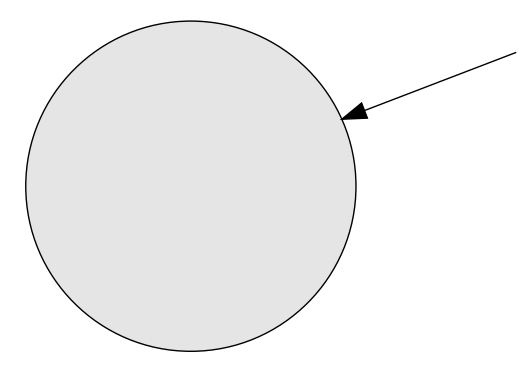


Section 5 gives lower bounds on the number of probes needed to solve these problems. Section 6 summarizes and suggests directions for future work.

\section{Definitions, Notation, and Assumptions}

In this section, we introduce definitions and notation used throughout the remainder of this paper, and state the assumptions we make on the object being tested. For the most part, notation and definitions are consistent with $[12,17]$.

For a point $p$, we use the notation $\mathrm{x}(p), \mathrm{y}(p)$, and $\mathrm{z}(p)$ to denote the $x, y$, and $z$ coordinates of $p$, respectively. The symbol $o_{+}$is used to denote the origin of the coordinate system. We use the notation dist $(a, b)$ to denote Euclidean distance between two objects. If $A$ and $B$ are sets of points, $\operatorname{dist}(A, B)$ is the minimum distance between all pairs of points in $A$ and $B$, i.e.,

$$
\operatorname{dist}(A, B)=\min \{\operatorname{dist}(a, b): a \in A, b \in B\}
$$

The angle formed by three points $a, b$, and $c$, is denoted by $\angle a b c$, and we always mean the smaller angle unless stated otherwise.

An object $I$ is defined to be any compact simply connected subset of $\mathrm{R}^{2}$ or $\mathrm{R}^{3}$ (it will be clear from the context), with boundary denoted by $\mathrm{bd}(I)$. For a point $p$, we use $R(p, I)$ and $r(p, I)$ to denote the maximal and minimal distance, respectively, from $p$ to a point in $\operatorname{bd}(I)$. I.e.,

$$
\begin{aligned}
R(p, I) & =\max \left\{\operatorname{dist}\left(p, p^{\prime}\right): p^{\prime} \in \operatorname{bd}(I)\right\} \\
r(p, I) & =\min \left\{\operatorname{dist}\left(p, p^{\prime}\right): p^{\prime} \in \operatorname{bd}(I)\right\} .
\end{aligned}
$$

For a point $p$, let

$$
\operatorname{qual}(p, I)=\min \{r(p, I)-(1-\epsilon),(1+\epsilon)-R(p, I)\}
$$

and let

$$
\operatorname{qual}(I)=\max \left\{\operatorname{qual}(p, I): p \in \mathbb{R}^{2}\right\}
$$

Any point $c_{I}$ with qual $\left(c_{I}, I\right)=$ qual $(I)$ is called a center of $I$. The value qual $(I)$ is called the quality of the object $I$, since it measures the maximum deviation of $I$ from a disk (or ball) of unit radius. An object $I$ with qual $(I)>0$ is $\operatorname{good}$ while an object $I$ with qual $(I)<0$ is bad. A procedure that determines whether a planar object is good or bad is called a roundness classification procedure. 
In order to have a testing procedure that is always correct and that terminates, it is necessary to make some assumptions about the object $I$ being tested. The following assumption made in $[12,17]$ is referred to as the minimum quality assumption, and refers to the fact that the manufacturing process can guarantee that manufactured objects have a minimum quality (although perhaps not enough to satisfy our roundness criterion).

Assumption 1. $R\left(c_{I}, I\right) \leq 1+\delta$ and $r\left(c_{I}, I\right) \geq 1-\delta$, for some constant $0<\delta<1 / 30$, i.e., the boundary of $I$ is contained in an annulus of inner radius $1-\delta$ and outer radius $1+\delta$.

The minimum quality assumption alone is not sufficient. If the object under consideration contains oddly shaped recesses, then it may be the case that these recesses cannot be found using finger probes. We say that an object $I$ is star-shaped if there exists a point $k \in I$ such that for any point $p \in I$, the line segment joining $k$ and $p$ is a subset of $I$. We call the set of all points with this property the kernel of $I$. The following assumption ensures that all points in $\operatorname{bd}(I)$ can be probed by directing probes close to a center of $I$.

Assumption 2. $I$ is a star-shaped object, and its kernel contains all points $p$ such that $\operatorname{dist}\left(c_{I}, p\right) \leq$ $\alpha$, for some constant $\alpha>2 \delta$.

In $[12,17]$, a roundness testing procedure is described that requires only Assumption 1 and the assumption that $I$ is convex. We observe that our assumptions are weaker.

Observation 1. The set of convex objects satisfying Assumption 1 is strictly contained in the set of objects satisfying Assumptions 1 and 2.

To see this, one need only observe that every convex object is a star-shaped object whose kernel is itself. Therefore every convex object satisfying Assumption 1 satisfies Assumption 2 with $\alpha=1-\delta>2 \delta$.

\section{Testing Disks}

In this section we give a procedure for testing the quality of a planar object $I$. We begin by describing a simplified procedure that assumes a center of $I$ is known to be the origin, $o_{+}$. The motivation for describing this simplified procedure is pedagogical. It is a simple example that helps in understanding the full procedure. 


\subsection{The Simplified Procedure}

Pseudocode for the testing proceudure is given in Procedure 1. It tests the roundness of an object $I$ by taking a set $S$ of probes at uniform intervals directed at the origin. Throughout this section, we use the notation $\operatorname{probe}(n, p)$ to denote the set of points obtained by taking $n$ probes directed at the point $p$ in directions $2 \pi / n, 4 \pi / n, \ldots, 2(n-1) \pi / n$. The procedure repeatedly doubles the size of the sample until either (1) a set of sample points is found that cannot be covered by an annulus of inner radius $1-\epsilon$ and outer radius $1+\epsilon$, in which case $I$ is rejected, or (2) the set of sample points can be covered by an annulus with inner radius sufficiently larger than $1-\epsilon$ and outer radius sufficiently smaller than $1+\epsilon$, in which case we can be sure that $I$ is a good object.

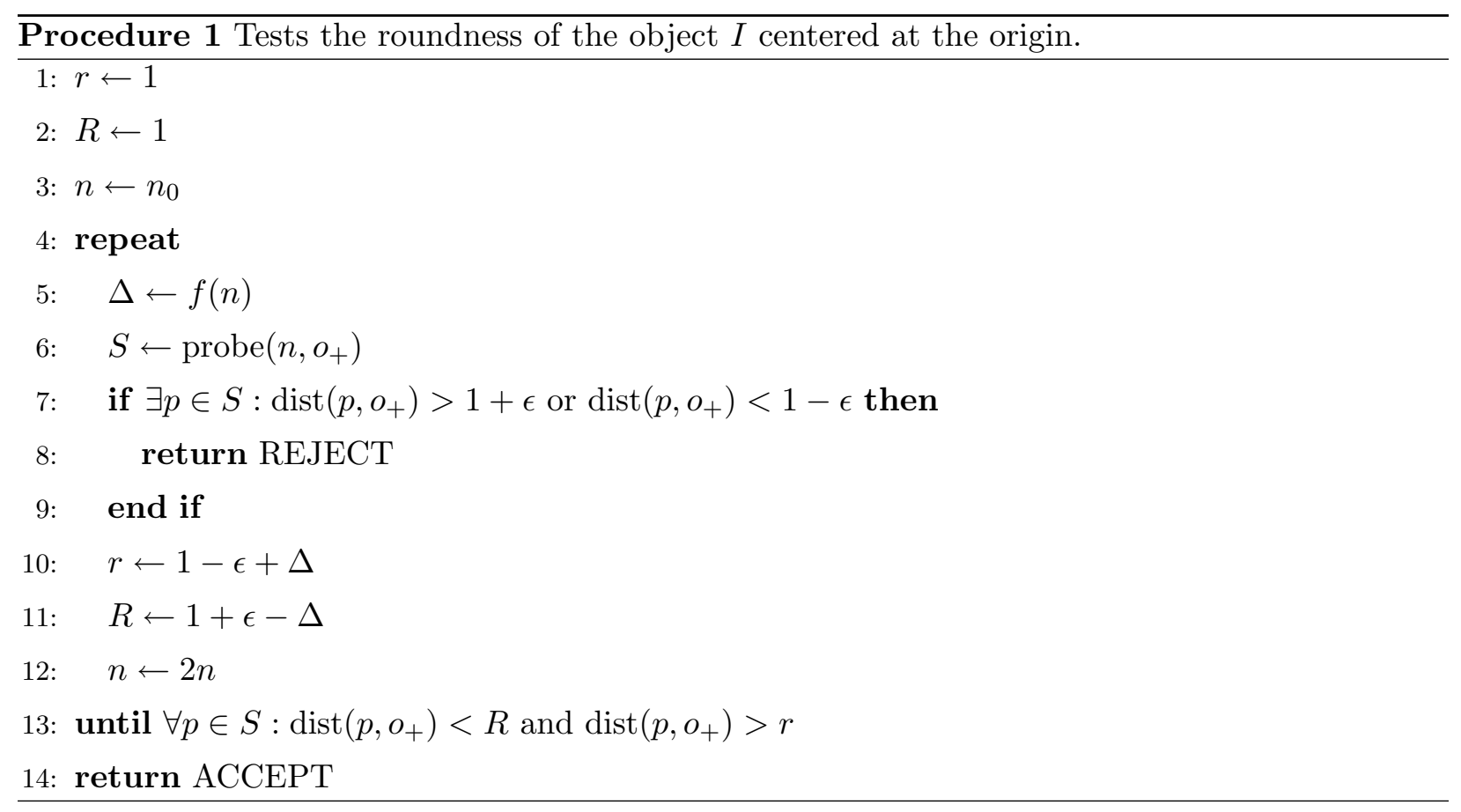

The function $f(n)$ that appears in the pseudocode is defined as

$$
f(n)=\sqrt{\left(\frac{(1+\delta) \pi}{n}\right)^{2}+\left(\frac{(1+\delta)^{2} \pi}{\alpha n}\right)^{2}}=O(1 / n),
$$

for any constant $\alpha$ and the constant $n_{0}$ is defined as

$$
n_{0}=\lceil\pi / \arctan (\alpha /(1+\delta))\rceil=O(1)
$$

With these definitions, we obtain the following crucial lemma.

Lemma 1. Let $I$ be a planar object with center $c_{I}$ and satisfying Assumptions 1 and 2. Let $S$ be 
the set of results of $n \geq n_{0}$ probes directed at $c_{I}$ in directions $0,2 \pi / n, 4 \pi / n, \ldots, 2 \pi(n-1) / n$. Then for any point $p \in \operatorname{bd}(I)$, there exists a point $p^{\prime} \in S$ such that $\operatorname{dist}\left(p, p^{\prime}\right) \leq f(n)$.

Proof. Assume wlog that $c_{I}=o_{+}, \mathrm{x}(p)=0$, and $1-\delta \leq \mathrm{y}(p) \leq 1+\delta$. We will upper-bound $\left|\mathrm{x}(p)-\mathrm{x}\left(p^{\prime}\right)\right|$ and $\left|\mathrm{y}(p)-\mathrm{y}\left(p^{\prime}\right)\right|$. Refer to Figure 3 for an illustration.

First note that there exists a sample point $p^{\prime} \in S$ such that $0 \leq \angle p c_{I} p^{\prime} \leq \pi / n$. By Assumption 1 , $\operatorname{dist}\left(o_{+}, p^{\prime}\right) \leq 1+\delta$, so an upper bound on $\left|\mathrm{x}(p)-\mathrm{x}\left(p^{\prime}\right)\right|$ is

$$
\begin{aligned}
\left|\mathrm{x}(p)-\mathrm{x}\left(p^{\prime}\right)\right|=\left|\mathrm{x}\left(p^{\prime}\right)\right| & \leq(1+\delta) \sin (\pi / n) \\
& \leq(1+\delta) \pi / n
\end{aligned}
$$

Since $\angle p c_{I} p^{\prime} \leq \pi / n, p^{\prime}$ must lie in the cone defined by the inequality

$$
\mathrm{y}\left(p^{\prime}\right) \geq\left|\mathrm{x}\left(p^{\prime}\right)\right|\left(\frac{\cos (\pi / n)}{\sin (\pi / n)}\right) .
$$

Next we note that the slope of the line through $p^{\prime}$ and $p$ must be in the range $[-\mathrm{y}(p) / \alpha, \mathrm{y}(p) / \alpha]$, otherwise Assumption 2 is violated. If $n \geq n_{0}$, then the region in which $p^{\prime}$ can be placed is bounded, and $\left|\mathrm{y}(p)-\mathrm{y}\left(p^{\prime}\right)\right|$ is maximized when $p^{\prime}$ lies on one of the bounding lines

$$
\begin{aligned}
& f_{l}(x)=x y(p) / \alpha+\mathrm{y}(p) \\
& f_{r}(x)=-x y(p) / \alpha+\mathrm{y}(p)
\end{aligned}
$$

Since both lines are symmetric about $x=0$ we can assume that $\mathrm{x}\left(p^{\prime}\right)$ lies on $f_{l}$, giving

$$
\begin{aligned}
\left|\mathrm{y}(p)-\mathrm{y}\left(p^{\prime}\right)\right| & \leq\left|\mathrm{y}(p)-f_{l}\left(\mathrm{x}\left(p^{\prime}\right)\right)\right| \\
& =\left|\mathrm{x}\left(p^{\prime}\right) \mathrm{y}(p) / \alpha\right| \\
& \leq\left|\mathrm{x}\left(p^{\prime}\right)(1+\delta) / \alpha\right| \\
& \leq(1+\delta)^{2} \pi / \alpha n
\end{aligned}
$$

Substituting (9) and (16) into the Euclidean distance formula and simplifying yields the stated inequality.

Theorem 1. There exists a roundness classification procedure that can correctly classify any planar object $I$ with center $c_{I}=o_{+}$and satisfying Assumptions 1 and 2 using $O(\mid 1 /$ qual $(I) \mid)$ probes and $O(\mid 1 /$ qual $(I) \mid)$ computation time. 


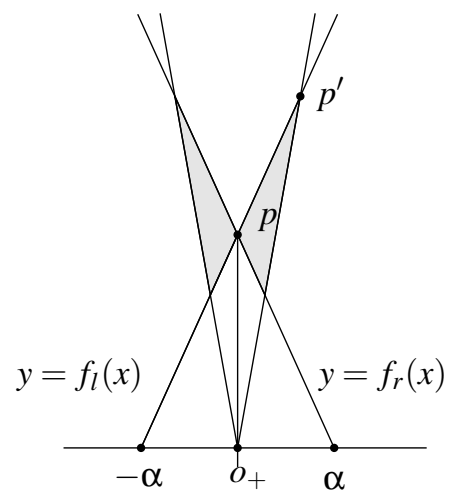


Proof. We begin by showing that the procedure is correct. We need to show that the procedure never rejects a good object and never accepts a bad object. The former follows from the fact that the procedure only ever rejects an object when it finds a point on the object's boundary that is not contained in the annulus of inner radius $1-\epsilon$ and outer radius $1+\epsilon$ centered at $c_{I}$.

Next we prove that the procedure never accepts a bad object. Lemma 1 shows that there is no point in $\operatorname{bd}(I)$ that is of distance greater than $f(n)$ from all points in $S$. The procedure only accepts $I$ when all points in $S$ are of distance at least $\Delta=f(n)$ from the boundary of the annulus of inner radius $1-\epsilon$ and outer radius $1+\epsilon$ centered at $o_{+}$. Therefore, if the procedure accepts $I$, all points in $\operatorname{bd}(I)$ are contained in an annulus of inner radius $1-\epsilon$ and outer radius $1+\epsilon$, i.e., the object is good.

Next we prove that the running time is $O(\mid 1 /$ qual $(I) \mid)$. First we observe that $f(n) \in O(1 / n)$. Next, note that the computation time and number of probes used during each iteration is linear with respect to the value of $n$, and the value of $n$ doubles after each iteration. Thus, asymptotically, the computation time and number of probes used are dominated by the value of $n$ during the last iteration. There are two cases to consider.

Case 1: Procedure 1 accepts $I$. In this case, the procedure will certainly terminate once $\Delta \leq$ qual $(I)$. This takes $O(\log (1 /$ qual $(I)))$ iterations. During the final iteration, $n \in O(1 /$ qual $(I))$.

Case 2: Procedure 1 rejects $I$. In this case, there is a point on $\operatorname{bd}(I)$ at distance qual $(I)$ outside the circle with radius $1+\epsilon$ centered at $o_{+}$, or there is a point in $\operatorname{bd}(I)$ at distance qual $(I)$ inside of the circle with radius $1-\epsilon$ centered at $o_{+}$. In either case, Lemma 1 ensures that the procedure will find a bad point within $O(\log \mid 1 /$ qual $(I) \mid)$ iterations. During the final iteration, $n \in O(\mid 1 /$ qual $(I) \mid)$.

\subsection{The Full Procedure}

The difficulty in implementing Procedure 1 is that we may not know the position of an exact center, $c_{I}$, of $I$. However, the following result from $[12,17]$ allows us to use this procedure anyhow.

Theorem 2 (Near-Center). Let I be a planar object with center $c_{I}$ and that satisfies Assumption 1. Then 6 probes and constant computation time suffice to determine a point $c_{0}$ such that $\operatorname{dist}\left(c_{I}, c_{0}\right) \leq 2 \delta$.

We call any such point $c_{0}$ a near-center of $I$. As the following lemmata show, knowing a near center is almost as useful as knowing a true center. Before we state the lemma, we need the following 
definitions.

$$
\begin{aligned}
g(n) & =\sqrt{\left(\frac{\pi(1+3 \delta)}{n}\right)^{2}+\left(\frac{\pi(1+3 \delta)^{2}}{n(\alpha-2 \delta)}\right)^{2}} \\
n_{0}^{\prime} & =\lceil\pi / \arctan (\alpha /(1+3 \delta))\rceil
\end{aligned}
$$

The value of $g(n)$ and $n_{0}^{\prime}$ are modifications of $f^{\prime}(n)$ and $n_{0}$ that are required to compensate for the fact that $c_{0}$ is a near center rather than a center.

Lemma 2. Let I be a planar object with center $c_{I}$, near-center $c_{0}$ and satisfying Assumptions 1 and 2. Let $S$ be the set of results of $n \geq n_{0}^{\prime}$ probes directed at $c_{0}$ in directions $0,2 \pi / n, 4 \pi / n, \ldots, 2 \pi(n-$ $1) / n$. Then for any point $p \in \operatorname{bd}(I)$, there exists a point $p^{\prime} \in S$ such that $\operatorname{dist}\left(p, p^{\prime}\right) \leq g(n)$.

Proof. The proof is almost a verbatim translation of the proof of Lemma 1, except that we assume that $c_{0}=o_{+}$. With this assumption we derive the bounds

$$
\begin{aligned}
&\left|\mathrm{x}(p)-\mathrm{x}\left(p^{\prime}\right)\right| \leq(1+3 \delta)(\pi / n) \\
&\left|\mathrm{y}(p)-\mathrm{y}\left(p^{\prime}\right)\right| \leq(1+3 \delta)^{2} \pi / n(\alpha-2 \delta) \mid
\end{aligned}
$$

Substituting these values into the formula for the Euclidean distance and simplifying yields the desired result.

Lemma 3. Let $I$ be a planar object with center $c_{I}$, near-center $c_{0}$ and satisfying Assumptions 1 and 2. Let $S$ be the set of results of $n$ probes directed at $c_{0}$ in directions $0,2 \pi / n, 4 \pi / n, \ldots, 2 \pi(n-1) / n$, and let $c_{S}$ be the center of $S$. Then

$$
\begin{aligned}
R\left(c_{S}, S\right) & \leq R\left(c_{S}, I\right) \leq R\left(c_{S}, S\right)+g(n) \\
r\left(c_{S}, S\right)-g(n) & \leq r\left(c_{S}, I\right) \leq r\left(c_{S}, S\right) .
\end{aligned}
$$

Proof. We prove only the bounds on the $R\left(c_{S}, I\right)$ as the proof of the bounds on $r\left(c_{S}, I\right)$ are symmetric. The lower bound on $R\left(c_{S}, I\right)$ is immediate, since $S \subset \operatorname{bd}(I)$. To see the upper bound, choose any point $p \in \operatorname{bd}(I)$ such that $\operatorname{dist}\left(c_{S}, p\right)=R\left(c_{S}, I\right)$. By Lemma 2 there exists $p^{\prime} \in S$ such that $\operatorname{dist}\left(p^{\prime}, p\right) \leq g(n)$. Therefore $\operatorname{dist}\left(c_{S}, p\right) \leq \operatorname{dist}\left(c_{S}, p^{\prime}\right)+g(n)$, which implies that $R\left(c_{S}, I\right) \leq R\left(c_{S}, S\right)+g(n)$.

Lemma 4. Let I be a planar object with center $c_{I}$, near-center $c_{0}$ and satisfying Assumptions 1 and 2. Let $S$ be the set of results of $n$ probes directed at $c_{0}$ in directions $0,2 \pi / n, 4 \pi / n, \ldots, 2 \pi(n-1) / n$. Then qual $(S)-g(n) \leq$ qual $(I) \leq \operatorname{qual}(S)$ 
Proof. qual $(I) \leq$ qual $(S)$ follows immediately from the fact that $S \subset \operatorname{bd}(I)$. For the lower bound, observe,

$$
\begin{aligned}
\operatorname{qual}(I) & =\max _{p \in \mathbb{R}^{2}} \operatorname{qual}(p, I) \\
& \geq \operatorname{qual}\left(c_{S}, I\right) \\
& =\min \left\{r\left(c_{S}, I\right)-(1-\epsilon),(1+\epsilon)-R\left(c_{S}, I\right)\right\} \\
& \geq \min \left\{r\left(c_{S}, S\right)-g(n)-(1-\epsilon),(1+\epsilon)-\left(R\left(c_{S}, S\right)+g(n)\right)\right\} \\
& =\min \left\{r\left(c_{S}, S\right)-(1-\epsilon),(1+\epsilon)-R\left(c_{S}, S\right)\right\}-g(n) \\
& =\operatorname{qual}(S)-g(n) .
\end{aligned}
$$

Theorem 3. There exists a roundness classification procedure that can correctly classify any planar object I satisfying Assumptions 1 and 2 using $O(1 / \mid$ qual $(I) \mid)$ probes and $O(\mid 1 /$ qual $(I)|\log | 1 /$ qual $(I) \mid)$ computation time.

Proof. We make the following modifications to Procedure 1. In Line 3, we set the value of $n$ to $n_{0}^{\prime}$. In Line 5 , we replace $f(n)$ with $g(n)$. In Line 6 we direct our probes at $c_{0}$ rather than $o_{+}$. In Lines 7 and 13, we replace the simple test with a call to one of the $O(n \log n)$ time referenced roundness algorithms in [4] or [7], to test whether the sample set $S$ can be covered by an annulus with the specified inner and outer radius.

Lemma 4 ensures that the procedure never accepts a bad object and never rejects a good object. i.e., the procedure is correct. The procedure terminates once $g(n)<\mid$ qual $(I) \mid$. This happens after $O(\log \mid 1 /$ qual $(I) \mid)$ iterations, at which point $n \in O(\mid 1 /$ qual $(I) \mid$.

\section{Testing Balls}

In this section we describe a complete procedure for testing the quality of manufactured balls. Since the first step in the algorithm is finding a point that is close to a center of our object, we begin by giving a procedure that can find such a point using a constant number of probes. 


\subsection{Finding a Near-Center}

In this section we describe a procedure for finding a point close to a center, $c_{I}$, of $I$. A near-center of $I$ is any point $c_{0}$ such that $\operatorname{dist}\left(c_{0}, c_{I}\right) \leq 2 \delta$. Our procedure uses three simple subroutines $X(p)$, $Y(p)$ and $Z(p)$. These subroutines perform two probes directed at $p$. The two probes come from opposite directions, and are parallel to the $x, y$, and $z$ axes, respectively. If the two probes contact $I$ at points $a$ and $b$, then the subroutines return $(a+b) / 2$, i.e., the midpoint between $a$ and $b$. If the probes do not contact $I$ then the routines return the point $p$. Pseudocode is given in Procedure 2 .

Procedure 2 Returns a near-center given a point $p_{0} \in I$.
1: $p_{1} \leftarrow X\left(p_{0}\right)$
2: $p_{2} \leftarrow Y\left(p_{1}\right)$
3: $p_{3} \leftarrow Z\left(p_{2}\right)$
4: $p_{4} \leftarrow X\left(p_{3}\right)$
5: $p_{5} \leftarrow Y\left(p_{4}\right)$
6: return $p_{5}$

Theorem 4. Let $I$ be an object with center $c_{I}$ and satisfying Assumption 1. Then 10 probes and constant computation time suffice to find a point $c_{0}$ such that $\operatorname{dist}\left(c_{I}, c_{0}\right) \leq 2 \delta$.

Proof. We will incrementally refine the bounds on the $x, y$, and $z$ coordinates of $p_{1}-p_{5}$. Refer to Figure 4 for illustrations. When using the terms left, right, up, down, vertical and horizontal, we will do so with respect to the relevant part of Figure 4.

Assume wlog that $c_{I}=o_{+}$. First we show that

$$
\left|\mathrm{x}\left(p_{1}\right)\right| \leq 2 \sqrt{\delta}
$$

Let $L_{1}$ be the line $y=\mathrm{y}\left(p_{0}\right), z=\mathrm{z}\left(p_{0}\right)$, i.e., the line along which the first two probes are taken. Consider the intersection of the plane that contains both $L_{1}$ and $c_{I}$ with $I$ (Figure 4 (a)). We use the notation $\mathrm{y}^{\prime}(p)$ to denote the distance of the point $p$ from the $x$-axis. There are two cases to consider.

Case 1: $\left|\mathrm{y}^{\prime}\left(p_{0}\right)\right| \leq 1-\delta$. Let $v_{l}$ and $v_{r}$ be the contact points of the left and right probes, respectively. Since the situation is symmetric about the $y^{\prime}$-axis, we bound $\left|\mathrm{x}\left(p_{1}\right)\right|=\left|\left(\mathrm{x}\left(v_{l}\right)+\mathrm{x}\left(v_{r}\right)\right) / 2\right|$ by maximizing both $\mathrm{x}\left(v_{l}\right)$ and $\mathrm{x}\left(v_{r}\right)$. Using the Pythagorean theorem and Assumption 1, we obtain

$$
\mathrm{x}\left(v_{l}\right)+\mathrm{x}\left(v_{r}\right)=\left(\operatorname{dist}\left(v_{r}, c_{I}\right)^{2}-\mathrm{y}^{\prime}\left(p_{0}\right)^{2}\right)^{\frac{1}{2}}-\left(\operatorname{dist}\left(v_{l}, c_{I}\right)^{2}-\mathrm{y}^{\prime}\left(p_{0}\right)^{2}\right)^{\frac{1}{2}}
$$




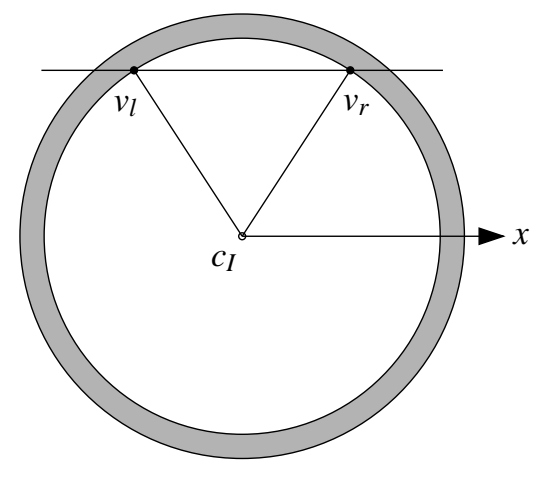




$$
\begin{aligned}
& \leq\left((1+\delta)^{2}-\mathrm{y}^{\prime}\left(p_{0}\right)^{2}\right)^{\frac{1}{2}}-\left((1-\delta)^{2}-\mathrm{y}^{\prime}\left(p_{0}\right)^{2}\right)^{\frac{1}{2}} \\
& =\frac{(1+\delta)^{2}-\mathrm{y}^{\prime}\left(p_{0}\right)^{2}-\left((1-\delta)^{2}-\mathrm{y}^{\prime}\left(p_{0}\right)^{2}\right)}{\left((1+\delta)^{2}-\mathrm{y}^{\prime}\left(p_{0}\right)^{2}\right)^{\frac{1}{2}}+\left((1-\delta)^{2}-\mathrm{y}^{\prime}\left(p_{0}\right)^{2}\right)^{\frac{1}{2}}} \\
& =\frac{4 \delta}{\left((1+\delta)^{2}-\mathrm{y}^{\prime}\left(p_{0}\right)^{2}\right)^{\frac{1}{2}}+\left((1-\delta)^{2}-\mathrm{y}^{\prime}\left(p_{0}\right)^{2}\right)^{\frac{1}{2}}} \\
& \leq \frac{4 \delta}{\left((1+\delta)^{2}-(1-\delta)^{2}\right)^{\frac{1}{2}}+\left((1-\delta)^{2}-(1-\delta)^{2}\right)^{\frac{1}{2}}} \\
& =\frac{4 \delta}{\sqrt{4 \delta}} \\
& =2 \sqrt{\delta} .
\end{aligned}
$$

Therefore, $\left|\left(\mathrm{x}\left(v_{l}\right)+\mathrm{x}\left(v_{r}\right)\right) / 2\right| \leq \sqrt{\delta}$, implying (29).

Case 2: $\left|\mathrm{y}^{\prime}\left(p_{0}\right)\right|>1-\delta$. In this case, we can define $v_{l}$ and $v_{r}$ as above. Note that $\mid\left(\mathrm{x}\left(v_{l}\right)+\right.$ $\left.\mathrm{x}\left(v_{r}\right)\right) / 2 \mid \leq \max \left\{\left|\mathrm{x}\left(v_{l}\right)\right|,\left|\mathrm{x}\left(v_{r}\right)\right|\right\}$. By the Pythagorean theorem, we have

$$
\begin{aligned}
\max \left\{\left|\mathrm{x}\left(v_{l}\right)\right|,\left|\mathrm{x}\left(v_{r}\right)\right|\right\} & \leq\left((1+\delta)^{2}-\mathrm{y}^{\prime}\left(p_{0}\right)^{2}\right)^{\frac{1}{2}} \\
& \leq\left((1+\delta)^{2}-(1-\delta)^{2}\right)^{\frac{1}{2}} \\
& =2 \sqrt{\delta},
\end{aligned}
$$

which is the desired result.

Next we show that

$$
\left|\mathrm{y}\left(p_{2}\right)\right| \leq 2 \sqrt{\delta}
$$

There are two cases to consider:

Case 1: The two probes contact $I$. In this case the same analysis used to show (29) yields the desired result.

Case 2: The two probes do not contact $I$. Let $L_{2}$ be the line defined by $x=\mathrm{x}\left(p_{1}\right), z=\mathrm{z}\left(p_{1}\right)$, i.e., the line along which the second set of probes are taken. Consider the intersection of the plane containing $L_{2}$ and $c_{I}$ with $I$ (Figure $4(\mathrm{~b})$ ). The point $p_{2}$ cannot be contained in the ball of radius $1-\delta$ centered at $c_{I}$, or else the probes would have contacted $I$. Therefore the probes must be to the left of the left dashed line or to the right of the right dashed line. Note however that $p_{1}$ must be contained in the sphere of radius $1+\delta$ centered at $c_{I}$. Thus, as in Case 2 of (29), the Pythagorean theorem provides the desired bound.

Next we will bound $\operatorname{dist}\left(c_{I}, c_{0}\right)$ by giving bounds on $\left|\mathrm{z}\left(p_{3}\right)\right|,\left|\mathrm{x}\left(p_{4}\right)\right|$ and $\left|\mathrm{y}\left(p_{5}\right)\right|$. Let $L_{3}$ be the line defined by $x=\mathrm{x}\left(p_{2}\right), y=\mathrm{y}\left(p_{2}\right)$, i.e., the line along which the third pair of probes is taken. 
From (29) and (30) it follows that $\operatorname{dist}\left(L_{3}, c_{I}\right) \leq \sqrt{8 \delta}$ (Figure 4 (c)). Note that since $\delta \leq 1 / 30$, $\sqrt{8 \delta} \leq 1-\delta$, and therefore, by Assumption 1 the third pair of probes must contact the object $I$.

Now consider the intersection of the plane that contains $L_{3}$ and $o_{+}$with the object $I$ (Figure $4(\mathrm{~d})$ ). We will use the notation $\mathrm{y}^{\prime \prime}(p)$ to denote the distance of the point $p$ from the $z$-axis. Define $d_{3}=\sqrt{8 \delta}$, and a computation similar to the one used to obtain (29) (Case 1) yields

$$
\left|\mathrm{z}\left(p_{3}\right)\right| \leq \frac{2 \delta}{\left((1+\delta)^{2}-\left(d_{3}\right)^{2}\right)^{\frac{1}{2}}+\left((1-\delta)^{2}-\left(d_{3}\right)^{2}\right)^{\frac{1}{2}}} .
$$

By the same argument, define $d_{4}=\left(2 \sqrt{\delta}+\mathrm{z}\left(p_{3}\right)^{2}\right)^{\frac{1}{2}}$ and $d_{5}=\left(\mathrm{z}\left(p_{3}\right)^{2}+\mathrm{x}\left(p_{4}\right)^{2}\right)^{\frac{1}{2}}$, and we obtain

$$
\begin{aligned}
\left|\mathrm{x}\left(p_{4}\right)\right| & \leq \frac{2 \delta}{\left((1+\delta)^{2}-\left(d_{4}\right)^{2}\right)^{\frac{1}{2}}+\left((1-\delta)^{2}-\left(d_{4}\right)^{2}\right)^{\frac{1}{2}}} \\
\left|\mathrm{y}\left(p_{5}\right)\right| \leq & \leq \frac{2 \delta}{\left((1+\delta)^{2}-\left(d_{5}\right)^{2}\right)^{\frac{1}{2}}+\left((1-\delta)^{2}-\left(d_{5}\right)^{2}\right)^{\frac{1}{2}}} .
\end{aligned}
$$

Note that $\mathrm{z}\left(c_{0}\right)=\mathrm{z}\left(p_{3}\right), \mathrm{x}\left(c_{0}\right)=\mathrm{x}\left(p_{4}\right)$, and $\mathrm{y}\left(c_{0}\right)=\mathrm{y}\left(p_{5}\right)$, so

$$
\operatorname{dist}\left(c_{I}, c_{0}\right)=\left(\mathrm{x}\left(p_{4}\right)^{2}+\mathrm{y}\left(p_{5}\right)^{2}+\mathrm{z}\left(p_{3}\right)^{2}\right)^{\frac{1}{2}} .
$$

By expanding (34) and substituting $\delta \leq 1 / 30$ in the denominators of (31), (32), and (33), it is straightforward, but tedious, to verify that $\operatorname{dist}\left(c_{I}, c_{0}\right) \leq 2 \delta$. (We used Maple.)

\subsection{Testing Quality}

Once a center or near-center of $I$ is known, we can obtain an approximation of the surface of $I$ by directing probes at this (near) center. In this section, we first describe a strategy for directing probes at the (near) center. We then describe the entire quality testing procedure for the case when the center of $I$ is known in advance. Finally, we describe the procedure for the case when the center of $I$ is not known in advance. Proving the correctness of our procedures involves bounding the maximum error in our approximation of the surface.

\subsubsection{The Probing Strategy}

In this section, we describe a probing strategy for taking $\Theta\left(n^{2}\right)$ probes directed at a point $p$, where $n$ is an even positive integer. The strategy is designed so that for any direction $d$, there is a probe in some direction "not too far" from $d$. Refer to Figure 5 for an illustration of what follows. 


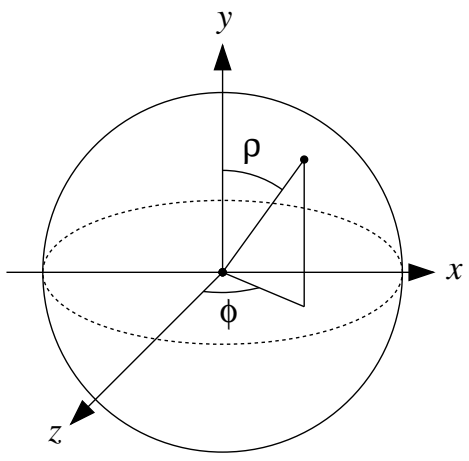


Consider the spherical coordinates $(\phi, \rho)$ of the unit sphere centered at $p$, where angles $\phi$ and $\rho$ are in the set $[0,2 \pi)$. We first divide the sphere into $n$ parallel slices, $s_{0}, \ldots, s_{n-1}$, such that slice $s_{i}$ contains all points where $\rho \in[i \pi / n,(i+1) \pi / n]$. Each slice $s_{i}$ is further subdivided into $m_{i}=$ $\lceil 2 n \max \{\sin (i \pi / n), \sin ((i+1) \pi / n)\}\rceil$ similar pieces, $s_{i 0}, \ldots, s_{i\left(m_{i}-1\right)}$, such that piece $s_{i j}$ contains all points in $s_{i}$ where $\phi \in\left[j \pi / m_{i},(j+1) \pi / m_{i}\right]$. We define the center of a piece $s_{i j}$ as the point with spherical coordinates $\left((2 i+1) \pi / 2 n,(2 j+1) \pi / 2 m_{i}\right)$.

Lemma 5. Let $a$ be any point in $s_{i j}$, and let $b$ be the center of $s_{i j}$. Then $\angle a p b \leq \pi / n$.

Proof. We begin by observing that the angle $\angle a p b$ is the length of the shortest path between $a$ and $b$ that remains on the surface of the unit sphere centered at $p$. We will proceed by constructing a two step path with the desired length.

The first step in our path is from $a$ to $(\phi(a), \rho(b))$. By construction of the slice $s_{i}$, we have $|\rho(a)-\rho(b)| \leq \pi / 2 n$. Hence the first step in the path is of length at most $\pi / 2 n$.

Now note that, since $n$ is even, the slice $s_{i}$ is the union of circles with radii in the range $[\sin (i \pi / n), \sin ((i+1) \pi / n)]$, and that one of these circles, call it $c$, contains both $(\phi(a), \rho(b))$ and $b$. The radius $r(c)$ of $c$ is at most $\max \{\sin (i \pi / n, \sin (i+1) \pi / n)\}$, therefore the piece $s_{i j}$ contains an arc of $c$ of length at most

$$
\begin{aligned}
2 \pi r(c) / m_{i} & \leq \frac{2 \pi \max \{\sin (i \pi / n), \sin ((i+1) \pi / n)\}}{\lceil 2 n \max \{\sin (i \pi / n), \sin ((i+1) \pi / n)\}\rceil} \\
& \leq \pi / n
\end{aligned}
$$

Since $b$ is in the center of this arc, the distance from $(\phi(a), \rho(b))$ to $b$ on $c$ is at most $\pi / 2 n$.

Our path from $a$ to $b$ stays on the surface of the unit sphere, and each of the two steps of our path is of length at most $\pi / 2 n$. This concludes the proof.

Lemma 6. $\sum_{i=0}^{n-1} m_{i} \in \Theta\left(n^{2}\right)$, i.e., the partitioning of the sphere described above contains $\Theta\left(n^{2}\right)$ pieces.

Proof. That the number of pieces is $O\left(n^{2}\right)$ follows from the inequality $\sin (\tau) \leq 1$. That the number of pieces is $\Omega\left(n^{2}\right)$ follows from the inequality $\sin (\tau) \geq 2 \tau / \pi$, for $\tau \in[0, \pi / 2]$.

For some center or near center $c$, our probing strategy involves directing probes along each of the half lines with an endpoint at $c$ and passing through the center of each piece of the sphere centered at $c$. In the remainder of the paper, we will use the notation probe $(n, c)$ to denote the set of probes obtained when using this strategy. 


\subsection{Measuring the Quality of Balls}

Given the method described in the previous section of taking probes, the remainder of the procedure for sampling is similar to the 2-dimensional procedure.

Lemma 7. Let $I$ be an object with center $c_{I}$, near-center $c_{0}$, and satisfying Assumptions 1 and 2. Let $S=\operatorname{probe}\left(n, c_{0}\right)$, for any $n \geq n_{0}^{\prime}$, where $\operatorname{dist}\left(c_{0}, c_{I}\right) \leq 2 \delta$. Then for any point $p \in \operatorname{bd}(I)$, there exists a point $p^{\prime} \in S$ such that $\operatorname{dist}\left(p, p^{\prime}\right) \leq g(n)$.

Proof. By Lemma 5, there exists a point $p^{\prime} \in S$ such that

$$
\angle p c_{I} p^{\prime} \leq \pi / n
$$

By orienting the coordinate system so that the plane $z=0$ passes through $p, p^{\prime}$ and $c_{I}$, we can assume, wlog, that

$$
\left|\mathrm{z}(p)-\mathrm{z}\left(p^{\prime}\right)\right|=0
$$

The remainder of the proof is identical to that of Lemma 2.

Theorem 5. There exists a roundness classification procedure that can correctly classify any object I satisfying Assumptions 1 and 2 using $O\left(1 /\right.$ qual $\left.(I)^{2}\right)$ probes and $O\left(1 /\right.$ qual $\left.(I)^{4}\right)$ computation time.

Proof. The proof of correctness and the bound on the number of probes follow by using exactly the same arguments used in Section 3. The bound on the computation time follows by using the algorithm of Duncan et al [7] that tests, in $O\left(n^{2}\right)$ time for a set of $n$ points in 3-dimensions, if there is a annulus with inner radius $1-\epsilon$ and outer radius $1+\epsilon$ that contains all the points (this is called the "referenced roundness decision problem").

\section{Lower Bounds}

In this section, we give lower bounds that show that the number of probes used in our testing procedures is optimal up to a constant factor. These lower bounds hold even if a center of the object is known in advance. The lower bounds use an adversary argument to show that, if a procedure uses too few probes then an adversary can orient a bad object so that its defects are "hidden" from all the probes, making the bad object indistinguishable from a similar good object.

We begin with a lower bound for planar objects. 
Theorem 6. Any roundness classification procedure that is always correct requires, in the worst case, $\Omega(\mid 1 /$ qual $(I) \mid)$ probes to classify a planar object $I$ with center $c_{I}=o_{+}$and satisfying Assumptions 1 and Assumption 2.

Proof. We prove the theorem by exhibiting two objects $I$ and $I^{\prime}$ with qual $(I)=\psi=-$ qual $\left(I^{\prime}\right)$, for any $0 \leq \psi \leq \epsilon$, such that $I$ and $I^{\prime}$ cannot be distinguished by any algorithm that uses $o(\mid 1 /$ qual $(I) \mid)$ probes.

The object $I$ is a perfect circle with radius $1-\epsilon+\psi$ and centered at the origin. The object $I^{\prime}$ is similar to $I$, except that it contains a conic recess of depth $4 \psi$ that removes an arc of length $8 \alpha \psi$ from the circle (see Figure 6). Note that qual $(I)=\psi$ and for $\alpha=1 / 9, \delta \leq 1 / 30$, and $\psi \leq \epsilon \leq \delta$, the assumptions of Section 2 hold for $I$ and $I^{\prime}$.

Furthermore, we claim that qual $\left(I^{\prime}\right)=-\psi$. To see this, let $p$ denote the apex of the recess and observe that the furthest point of $I^{\prime}$ from $p$ is at distance $2-2 \epsilon-2 \Psi$ (directly below $p$ in Figure 6 ). Therefore, any disk contained in $I^{\prime}$ must have all points within distance $2-2 \epsilon-2 \Psi$ of $p$, i.e., its radius is at most $1-\epsilon-\Psi$. Thus, any annulus that contains the boundary of $I^{\prime}$ must have inner radius at most $1-\epsilon-\Psi$, so qual $\left(I^{\prime}\right) \leq \Psi$. To see that qual $\left(I^{\prime}\right) \geq \Psi$ take an annulus whose center is $1-\epsilon-\Psi$ units below $p$ in Figure 6 .

Assume by way of contradiction that there exists a roundness classification procedure $\mathcal{P}$ that always accepts $I$ and always rejects $I^{\prime}$ using $n<2 \pi(1-\epsilon+\psi) / 8 \alpha \psi$ probes. Let $S$ be the set of $n$ probes made by $\mathcal{P}$ in classifying $I$. Since $|S|=n<2 \pi(1-\epsilon+\psi) / 8 \alpha \psi, S$ contains two probes, $p_{1}$ and $p_{2}$, that occur consecutively on $\operatorname{bd}(I)$, such that the length of $\operatorname{bd}(I)$ between $p_{1}$ and $p_{2}$ is greater than $8 \alpha \psi$. Note that we could place $I^{\prime}$ with its recess between $p_{1}$ and $p_{2}$, and the results of all the probes made by $\mathcal{P}$, and therefore the actions of $\mathcal{P}$, would be the same for $I$ and $I^{\prime}$. But this is a contradiction, since we assumed that $\mathcal{P}$ always correctly classifies both $I$ and $I^{\prime}$.

We conclude the proof by making the observation that $n=2 \pi(1-\epsilon+\psi) / 8 \alpha \psi \in \Omega(1 / \psi)=$ $\Omega(\mid 1 /$ qual $(I) \mid)=\Omega\left(\mid 1 /\right.$ qual $\left.\left(I^{\prime}\right) \mid\right)$.

For 3-dimensional objects we require the following lemma.

Lemma 8. Let $S$ be a set of $n^{2}$ points on the unit sphere. Then, there exists a spherical cap c with radius $1 / n$ such that $c$ contains no points of $S$

Proof. Consider the convex hull of $S$, which has at most $2 n^{2}-4$ faces. The plane passing through a face defines a spherical cap, and the union of these caps cover the entire sphere, a surface area of 

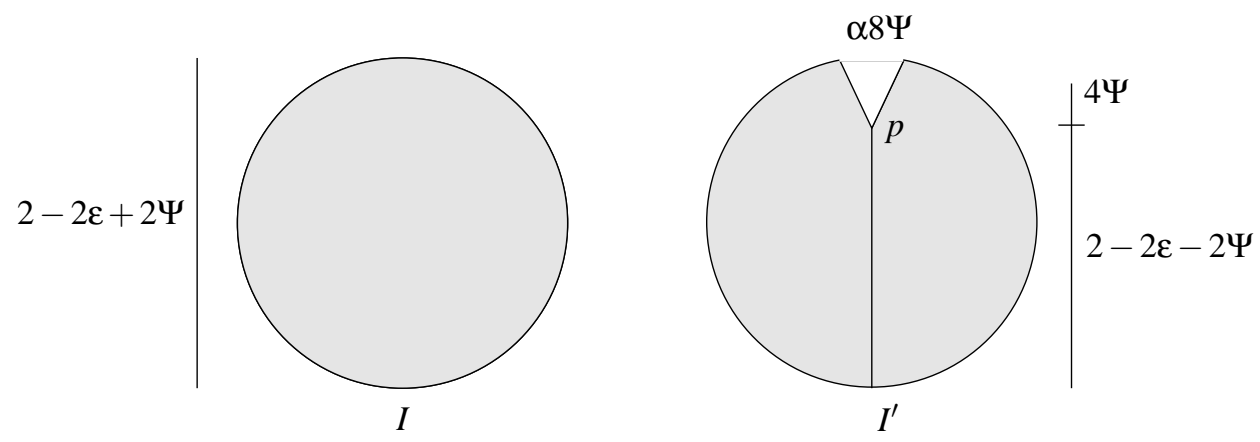
$4 \pi$. By the pigeonhole principle, some face $f$ must define a cap $c$ with surface area at least $2 \pi / n^{2}$. Furthermore, since $f$ is part of the convex hull, there are no other points of $S$ in this cap. The surface area of $c$ obeys the inequality sa $(c) \leq 2 \pi r^{2}$, where $r$ is the radius of $c$. Thus, we have the inequalities

$$
2 \pi r^{2} \geq \operatorname{sa}(c) \geq 2 \pi / n^{2}
$$

yielding $r \geq 1 / n$.

Theorem 7. Any roundness classification procedure that is always correct requires, in the worst case, $\Omega\left(\mid 1 /\right.$ qual $\left.(I)^{2} \mid\right)$ probes to classify a object $I$ with center $c_{I}=o_{+}$and satisfying Assumptions 1 and 2.

Proof. We proceed in almost the same way as in Theorem 6 . The object $I$ is a perfect ball with radius $1-\epsilon+\psi$. The object $I^{\prime}$ is similar to $I$, except that it contains a conic recess of depth $4 \psi$ that removes a circle of diameter $8 \alpha \psi$ from the surface of $I$.

Let $S$ be any set of $o\left(n^{2}\right)$ probes directed at $I$. Then, by Lemma 8, there exists a spherical cap $c$ on the surface of $I$ with radius $\omega(\psi)$ such that $c$ contains no point of $S$. We use the cap to hide the conic defect of $I^{\prime}$ so that the procedure cannot distinguish $I$ and $I^{\prime}$.

\section{Conclusions}

We have studied the problem of determining whether a manufactured disk or ball is sufficiently round. Our model for disks is less restrictive than that of $[12,17]$ yet our results are the same. Our result for balls is the first result in this area. We have also given lower bounds that show that our procedures are optimal in terms of the number of probes used.

Practitioners may question whether adaptive sampling methods such as those used in our roundness classification procedures are of wide spread usefulness, since production facilities are often arranged as "assembly lines" in which the each step in the assembly process should take a fixed, and known, amount of time. In this case, our results are still useful, since we can fix the number of probes used by our procedures. The bounds on the quality of the object can then be used to determine whether the object is definitely good, definitely bad, or unclear. A conservative production strategy can then simply reject objects that are bad or unclear. 


\section{Acknowledgement}

The authors would like to thank Silvia Götz for carefully reading several early revisions of this paper.

\section{References}

[1] P. K. Agarwal, B. Aronov, S. Har-Peled, and M. Sharir. Approximation and exact algorithms for minimum-width annuli and shells. In Proceedings of the 16th ACM Symposium on Computational Geometry (SoCG 99), pages 380-389, 1999.

[2] P. K. Agarwal, B. Aronov, and M. Sharir. Line transversals of balls and smallest enclosing cylinder in three dimensions. Discrete $\&$ Computational Geometry, 21:373-388, 1999.

[3] P. K. Agarwal, B. Aronov, and M. Sharir. Exact and approximation algorithms for minimumwidth cylindrical shells. Discrete \& Computational Geometry, 26, 2001.

[4] M. de Berg, P. Bose, D. Bremner, S. Ramaswami, and G. Wilfong. Computing constrained minimum-width annuli of point sets. Computer-Aided Design, 30:267-275, 1998.

[5] T. M. Chan. Approximating the diameter, width, smallest enclosing cylinder, and minimumwidth annulus. In Proceedings of the 17th ACM Symposium on Computational Geometry (SoCG 2000), pages 300-309, 2000.

[6] R. Cole and C. K. Yap. Shape from probing. Journal of Algorithms, 8:19-38, 1987.

[7] C. A. Duncan, M. T. Goodrich, and E. A. Ramos. Efficient approximation and optimization algorithms for computational metrology. In Proceedings of the 8th ACM-SIAM Symposium on Data Structures and Algorithms (SODA'9'7), pages 121-130, 1997.

[8] H. Ebara, N. Fukuyama, H. Nakano, and Y. Nakanishi. A practical algorithm for computing the roundness. IEICE Transactions on Information and Systems, E75:253-257, 1992.

[9] J. Garcia and P. A. Ramos. Fitting a set of points by a circle. Discrete $\&$ Computational Geometry, 20:389-402, 1998.

[10] R. Kumar and D. Sivakumar. Roundness estimation via random sampling. In Proceedings of the 10th ACM-SIAM Symposium on Data Structures and Algorithms (SODA'99), pages 603-612, 1999. 
[11] V. B. Le and D. T. Lee. Out-of-roundness problem revisited. IEEE Transactions on Pattern Analysis and Machine Intelligence, 13(3):217-223, 1991.

[12] K. Mehlhorn, T. C. Shermer, and C. K. Yap. A complete roundness classification procedure. In Proceedings of the 13th ACM Symposium on Computational Geometry (SoCG'97), pages 129-138, 1997.

[13] P. Ramos. Computing roundness in practice. In Proceedings of the 13th European Workshop on Computational Geometry, pages 125-126, 1997.

[14] P. A. Ramos. Computing roundness is easy if the set is almost round. In Proceedings of the 16th ACM Symposium on Computational Geometry (SoCG 99), pages 307-315, 1999.

[15] U. Roy and X. Zhang. Establishment of a pair of concentric circles with the minimum radial separation for assessing rounding error. Computer-Aided Design, 24(3):161-168, 1992.

[16] E. Schomer, J. Sellen, M. Teichmann, and C. K. Yap. Efficient algorithms for the smallest enclosing cylinder. In Proceedings of the 8th Canadian Conference on Computational Geometry, pages 264-269, 1996.

[17] T. Shermer and C. K. Yap. Probing for near-centers and estimating relative roundness. Presented at 1995 ASME Workshop on Tolerancing and Metrology, 1995.

[18] K. Swanson. An optimal algorithm for roundness determination on convex polygons. In Proceedings of the 3rd Workshop on Data Structures and Algorithms (WADS'93), pages 601609, 1993.

[19] C. K. Yap. Exact computational geometry and tolerancing metrology. In David Avis and Jit Bose, editors, Snapshots of Computational and Discrete Geometry, Vol. 3. Springer-Verlag, 1994. 